\title{
Inclusion of additional energy dissipation due to plunging breakers in parametric type wave models
}

\author{
Alireza Jafari $^{\dagger 1}$, Nick Cartwright ${ }^{\dagger}$, Amir Etemad-Shahidi $^{\dagger}$, Mahnaz Sedigh $^{\dagger}$ \\ †Griffith School of Engineering, Gold Coast Campus, Griffith University, Southport QLD 4215 Australia, a.jafari@ griffith.edu.au, \\ n.cartwright@griffith.edu.au, a.etemadshahidi@griffith.edu.au,m.sedigh@griffith.edu.au
}

\begin{abstract}
One of the most critical issues in coastal engineering problems is to accurately predict the wave height profile across the surfzone. Parametric wave models are broadly used in modelling the wave energy dissipation in this regard. Three parametric wave models based on the bore energy dissipation model were evaluated against new field measurements under various conditions from mild to stormy. The results indicate that a discrepancy between models and data occurred near the break point for cases where the wave breaking was of the plunging type but after the breakpoint the wave height decay rates compared well. In order to improve this model shortcoming, comprehensive new laboratory tests were conducted to quantify the additional energy dissipation due to plunging breakers. Based on the data, new empirical equations were derived and incorporated into the most recent bore dissipation parametric wave model. The inclusion of the extra energy dissipation due to plunging breakers results in significant improvement in the prediction of the wave height profile.
\end{abstract}

Keywords: Random waves, surfzone, wave transformation, wave breaking, energy dissipation, plunging breaker, bore dissipation, parametric wave model

\section{Introduction}

Wave energy modifies the beach and shoreline. In this interaction, the mechanical energy of wave transforms into heat. As this process is irreversible, it is called energy dissipation and a major proportion of energy loss occurs due to the wave breaking (Svendsen, 2006). However, the energy losses of incident waves due to mechanisms, such as bottom friction, percolation, and viscous loss, are also happening along with the wave breaking, but most of the time their values are negligible compare with dissipation due to breaking (Thornton and Guza, 1983).

In this regard, parametric wave propagation models are broadly used in many coastal engineering applications. Depending on the parameter fitting, they mainly reflect $80 \%-85 \%$ accuracy, which is desirable in most coastal area problems (Ruessink et al., 2003; van Rijn et al., 2003). However, errors

\footnotetext{
1 Corresponding author Tel.: +61 (07)55527608; fax: +61 (07) 555 27608. E-mail addresses: a.jafari@griffith.edu.au(A. Jafari), Postal Address: Griffith School of Engineering, Gold Coast Campus, Griffith University, QLD 4222, Australia
} 
in the wave prediction will propagate and accumulate into errors in the estimation of hydrodynamic related parameters such as wave set up, sediment transport and radiation stress (Guard and Baldock, 2007). Therefore, several researchers have put effort into this issue to increase the accuracy of parametric wave propagation models based on wave energy dissipation in a bore (e.g. Battjes and Janssen, 1978; Thornton and Guza, 1983; Baldock et al., 1998; Ruessink et al., 2003; Alsina and Baldock, 2007; Janssen and Battjes, 2007). Alsina and Baldock (2007) proposed a modified form of the parametric wave propagation model for a non-saturated surfzone based on Baldock et al. (1998). In the present study, Alsina and Baldock's (2007) model (hereafter referred to as AB07) is applied to field data collected in Southeast Queensland during both stormy and mild conditions (Jafari et al., 2011), as well as new laboratory data. Meanwhile, the model of Thornton and Guza (1983), hereafter referred to as TG83, and also Baldock et al. (1998), hereafter referred to as B98, are also evaluated against the data.

All of the above models are based on wave energy dissipation due to the classic bore model of Le Mahute (1962). This paper will demonstrate that a limitation of these models is the lack of consideration of additional energy dissipation that takes place during plunging type wave breaking. Based on new laboratory experiments, a new empirical formulation for the prediction of this extra plunging breaker dissipation is developed and incorporated into the AB07 model.

\section{Existing parametric wave models}

Battjes and Janssen (1978) took the very first step in this field and introduced their pioneer model which has since been modified and refined by others (e.g. TG83, B98, and AB07). Parametric models evaluate the wave height across the surfzone using energy flux equilibrium,

$$
\frac{\partial\left(E C_{g}\right)}{\partial x}=-D_{E}
$$

where, $C_{g}$ and $E$ are respectively the group velocity and wave energy which can be estimated using linear wave theory,

$$
\begin{aligned}
& E=\frac{1}{8} \rho g H_{r m s}^{2} \\
& C_{\mathrm{g}}=C \frac{1}{2}\left(1+\frac{2 k h}{\sinh 2 k h}\right) \cos \theta
\end{aligned}
$$

where $\rho$ is the water density, $C$ is the wave phase velocity, $k$ is the wave number, $h$ is the water depth, and $\theta$ is the approaching wave angle. $D_{E}$ denotes the time averaged wave energy dissipation which consists of primarily bore dissipation, $D_{B}$, and secondly dissipation due to bottom friction, $D_{f}$. In practice, however, friction effects are assumed to be negligible relative to those of wave breaking (Thornton and Guza, 1983; Baldock et al., 1998; Alsina and Baldock, 2007). 


\subsection{Bore dissipation for single wave}

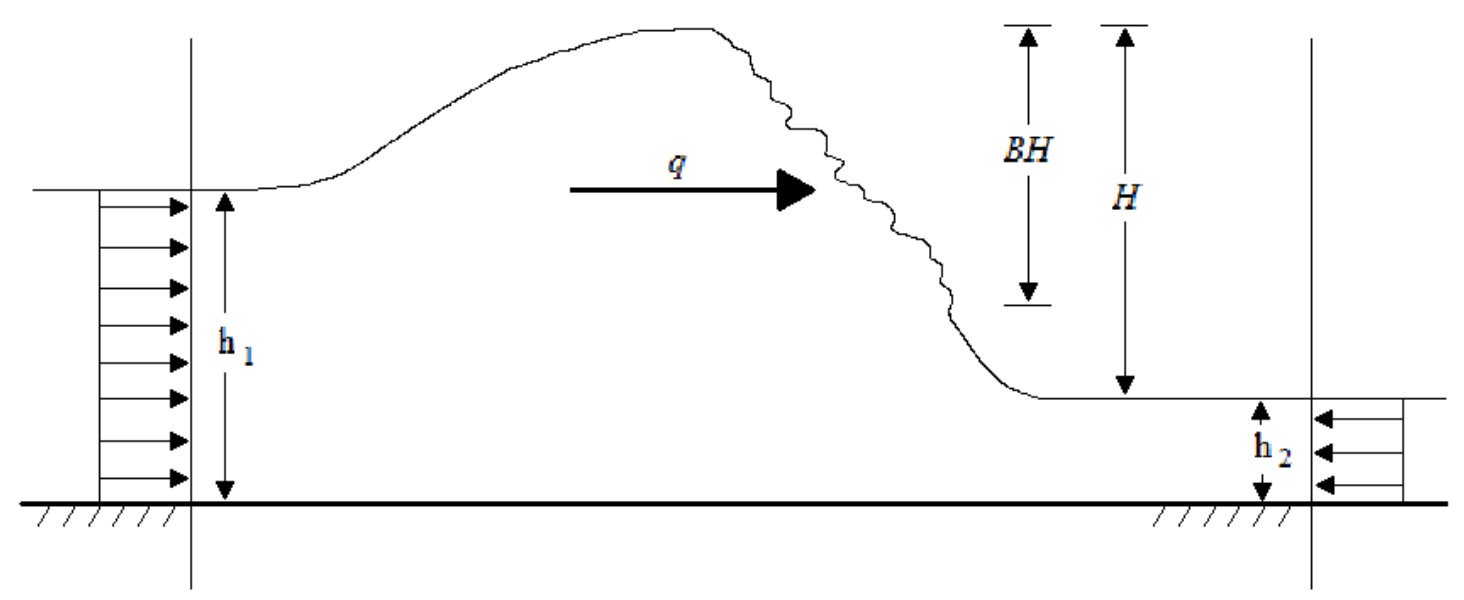

Figure 1. Schematic sketch of energy dissipation associated with a bore.

As a result of the wave breaking, the wave crest generally curls over and generates vortices at the water surface. The dissipation rate due to breaking depends on the size and strength of these vortices (Battjes and Janssen, 1978). The turbulence on the spilling breaker, qualitatively, is similar to a bore and can be treated as a travelling hydraulic jump (Figure 1). The bore dissipation for a single breaking wave can be calculated as follows (Thornton and Guza, 1983),

$$
D_{b}=\frac{1}{4} \rho g f_{p} \frac{(B H)^{3}}{h}
$$

\subsection{Bore dissipation for a wave distribution}

In order to obtain the average bore energy dissipation for a distribution of irregular waves, equation (5) needs to be multiplied by the number of breaking waves in the distribution. By integrating the product of equation (5) and the probability density function, Pdf, of the ratio of breaking waves over the total number of waves, parametric models evaluate the energy dissipation and wave transformation across the surfzone (Battjes and Janssen, 1978). The main difference between the various models rises from determining the fraction of breaking waves in an irregular sea wave state.

Batttjes and Janssen (1978) were pioneers in this area and used a truncated Rayleigh distribution with an implicit formula for the ratio of breaking waves over total number of waves, $Q_{b}$. After Batttjes and Janssen (1978), Thornton and Guza (1983) proposed an empirical weighted Rayleigh distribution, based on field data recorded from Torrey Pines beach. TG83 introduced two free parameters namely $B$ in single bore dissipation, i.e. equation (5), and $\gamma$ which is the ratio of wave height over water depth. These free parameters are used to calibrate the TG83 model. Consequently, achieving the best results from TG83 is limited to the availability of field data. 
B98 obtained the proportion of breaking waves, $Q_{b}$, directly from the Rayleigh distribution. $Q_{b}$ is determined by integrating the Rayleigh distribution over all waves for which $H / H_{r m s} \geq H_{b} / H_{r m s}$ resulting in (Baldock et al., 1998),

$$
Q_{b-B 98}=P\left\{H \geq H_{b}\right\}=\int_{H^{*}}^{\infty}\left\{\frac{2 H}{H_{r m s}} \exp \left[-\left(\frac{H}{H_{r m s}}\right)^{2}\right]\right\} d\left(\frac{H}{H_{r m s}}\right)=\exp \left[-\left(\frac{H_{b}}{H_{r m s}}\right)^{2}\right]
$$

where, $H^{*}=H_{b} / H_{r m s}$ and $H_{r m s}$ is the root mean square wave height and $H_{b}$ is maximum stable unbroken wave height (before the wave breaks) in a given water depth. B98 applied Nairn's (1990) expression of $H_{b}$ which is as follows,

$$
\frac{H_{b}}{h}=0.39+0.56 \tanh \left(33 S_{o}\right)
$$

where, $S_{o}$ is offshore wave steepness. B98 assumed that the relationship of $H / h$ in equation (5) is close to 1 . In addition, they suggested that factor $B$ can be considered 1 for simplification purposes and therefore eliminating a free parameter. Thus, the time averaged rate of energy dissipation proposed by B98 does not assume prior knowledge of the surfzone condition, which is given by (Baldock et al., 1998). The only free parameter that they used was $\gamma$. AB07 followed the same approach as B98. However they relaxed the underlying assumption that $H / h=1$ based on the field and laboratory data presented by Raubenheimer et al. (1996) which indicates that this is not the case close to shoreline. Hence, the original term of $\mathrm{H}^{3} / h$ in equation (5) was retained and AB07 proposed the following bore dissipation formula (Alsina and Baldock, 2007),

$$
\begin{aligned}
& D_{B-A B O 7}=D_{b} \times Q_{b}=\frac{1}{4} \rho g f_{p} B \frac{H_{r m s}^{3}}{h} \times \\
& \left\{\left[\left(\frac{H_{b}}{H_{r m s}}\right)^{3}+\frac{3}{2} \frac{H_{b}}{H_{r m s}}\right] \cdot \exp \left[-\left(\frac{H_{b}}{H_{r m s}}\right)^{2}\right]+\frac{3}{4} \sqrt{\pi}\left[1-\operatorname{erf}\left(\frac{H_{b}}{H_{r m s}}\right)\right]\right\}
\end{aligned}
$$

where, erf denote the error function. AB07 calculate the cut off depth at the shoreline as the rundown limit which estimated as $R_{d}=0.15 \times R_{u}$, where $R_{u}$ is the run-up vertical elevation defined as follows (Hunt, 1959),

$$
R_{u}=K_{r} \tan \beta \times \sqrt{H_{o} L_{o}}
$$

where $H_{o}$ and $L_{o}$, are the offshore wave height and wave length respectively, $K_{r}$ is a calibration constant which varies between 0.7 and 0.8 , and $\tan \beta$ being the beach slope. 


\section{Model - Data Comparison: Field data}

\subsection{Field data}

Field data was collected from a field site located at the Spit on the Gold Coast, Australia (Jafari et al., 2011). Table 1 presents the field conditions and wave statistical data sets used in the comparison against the AB07, B98, and TG83 models. All the recorded data were first checked and found to conform with the Rayleigh distribution (cf. Jafari and Cartwright, 2012).

Table 1. Field Condition tested against model prediction; where $S_{o}$ is offshore wave steepness, second column is representing the tide condition which MT-F stands for mid falling tide, LT stands for Low Tide, and HT stands for high tide

\begin{tabular}{ccccccccc}
\hline \hline Event & $\begin{array}{c}\text { Tidal } \\
\text { Stage }\end{array}$ & $\begin{array}{c}\text { Offshore } \\
\text { Boundary } \\
(\mathbf{m})\end{array}$ & $\begin{array}{c}\text { Offshore } \\
\text { Depth } \\
(\mathbf{m})\end{array}$ & $\begin{array}{c}\boldsymbol{H}_{\max } \\
(\mathbf{m})\end{array}$ & $\begin{array}{c}\boldsymbol{H}_{\text {rmso }} \\
(\mathbf{m})\end{array}$ & $\begin{array}{c}\boldsymbol{T}_{\boldsymbol{p}} \\
(\mathbf{S})\end{array}$ & $\begin{array}{c}\boldsymbol{T}_{\text {ave }} \\
(\mathbf{S})\end{array}$ & $\boldsymbol{S}_{\boldsymbol{o}}$ \\
\hline TC Hamish (11/03/2009) & MT-F & 500 & 7.6 & 4.8 & 2.3 & 8.2 & 8.2 & 0.022 \\
East Coast Lows (21/05/2009) & LT & 500 & 7.8 & 5.4 & 3.2 & 9.4 & 10.0 & 0.023 \\
East Coast Lows (21/05/2009) & LT & 300 & 5.6 & 2.7 & 1.1 & 9.7 & 12.0 & 0.008 \\
Mild Condition (11/11/2009) & LT & 500 & 5.4 & 0.9 & 0.6 & 7.9 & 7.2 & 0.008 \\
Mild Condition (11/11/2009) & LT & 300 & 3.4 & 2.1 & 0.8 & 7.9 & 6.1 & 0.013 \\
TC Ului (20/03/2010) & HT & 300 & 4.4 & 3.0 & 1.4 & 11.0 & 7.6 & 0.015 \\
\hline
\end{tabular}

\subsection{Results and Discussion}

Figure 2 shows the model data comparison for an East Coast low event with the model's offshore boundary set on $500 \mathrm{~m}$ tube length. It is clear that between $\mathrm{x}=500 \mathrm{~m}$ and $300 \mathrm{~m}$ the models are all missing some energy dissipation in this region. Unfortunately data is also lacking in this area but visual observations in the field on the day saw plunging waves breaking at $\mathrm{x} \sim 400 \mathrm{~m}$ (as judged relative to the adjacent jetty which extends ca. $300 \mathrm{~m}$ offshore). After the breakpoint (x $\sim 300 \mathrm{~m})$ the observed and predicted wave height profiles are essentially parallel to each other indicating that the bore dissipation model is adequate. To further illustrate this, the $\mathrm{x}=300 \mathrm{~m}$ data was set as the offshore boundary for the models (Figure 3). Model performance clearly improves however, a separation between models and data occurs at the second breaking point $(\mathrm{x} \sim 160 \mathrm{~m})$. Model-data comparisons for the other data sets show similar behaviour (cf. Baldock and Simmonds, 1999 for further details).

At this point it is therefore hypothesised that the observed model-data discrepancy near the break point (between $\mathrm{x}=500 \mathrm{~m}$ and $400 \mathrm{~m}$ ) is due to extra energy dissipation caused by plunging breakers which is not currently considered in the models. Due to the sparseness of the field data in the vicinity of the outer breakpoint it is not possible to accurately evaluate the extent of the extra energy dissipation (ie by numerically evaluating equation (1) for $\mathrm{D}_{\mathrm{E}}$ ) and so new laboratory experiments were undertaken. 

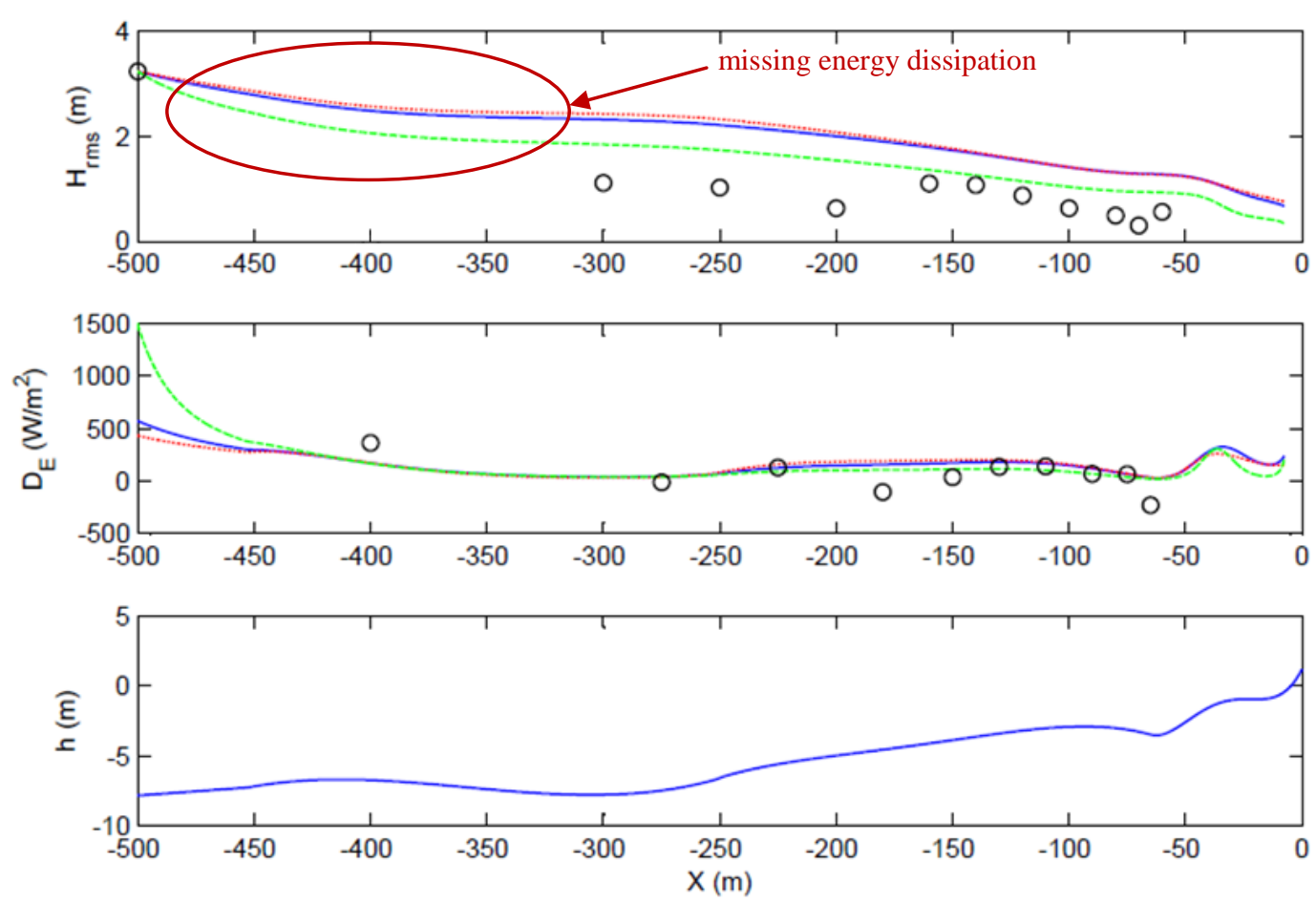

Figure 2. ECL $(500 \mathrm{~m})$ field data distribution along surfzone (circles) against model prediction of

AB07 (black solid line), B98 (black dotted line) and TG83 (gray dash line); top panel is $H_{r m s}$ distribution, 2nd panel is distribution of energy dissipation per unit area and 3rd panel is the beach profile. The red oval is indicating the area that energy dissipation is under estimated.
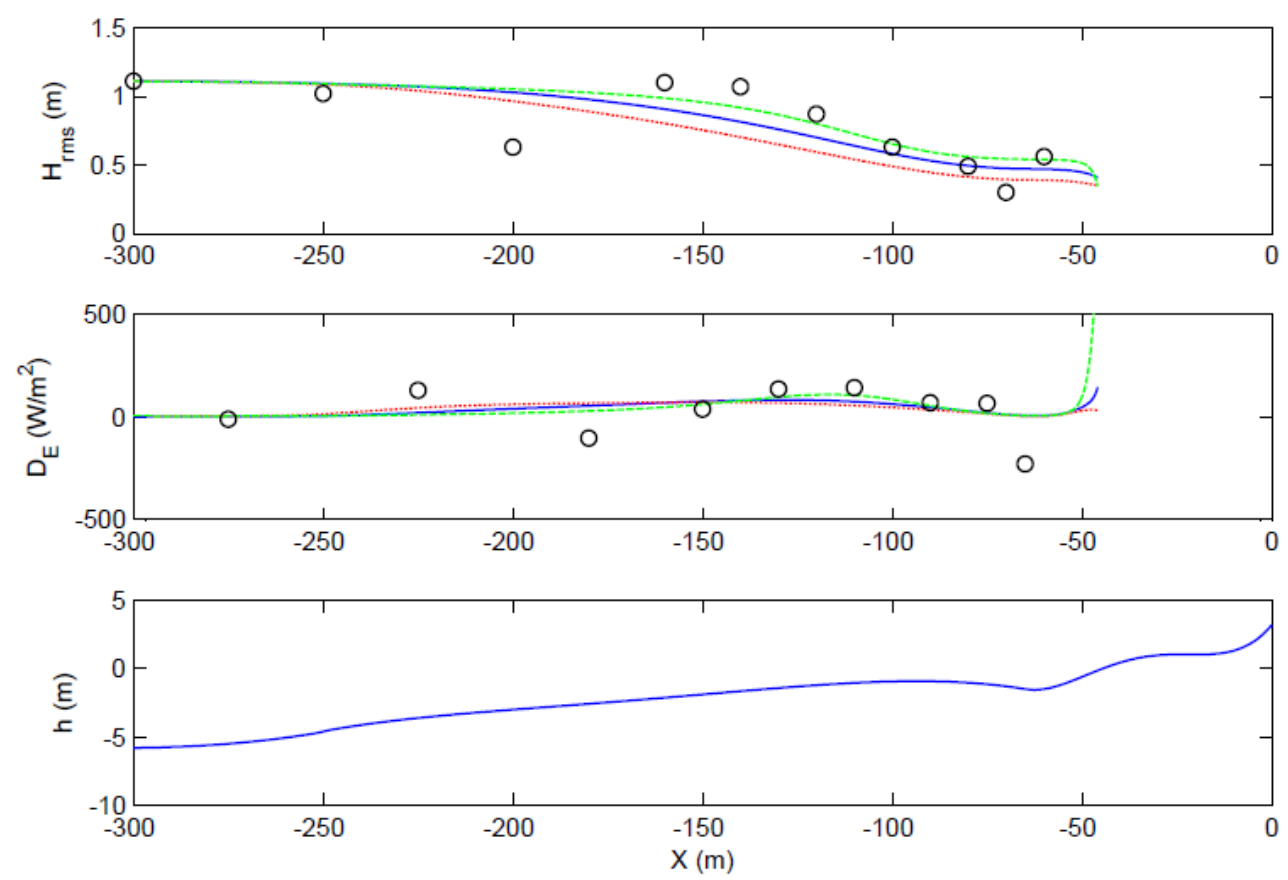

Figure 3. Model-data comparison using ECL (300 m) event. Top: $H_{r m s}$; 2nd panel: Energy dissipation per unit area: data (circless); AB07 (black solid line), B98 (black dotted line) and TG83 (gray dash line); Bottom panel: water depth profile 


\section{Model - Data Comparison: Laboratory data}

\subsection{Laboratory experiments}

The laboratory experiments were performed using an $8 \mathrm{~m}$ long and $0.5 \mathrm{~m}$ wide wave flume (Figure 4) with a 1:57 scale model of a typical Gold Coast bar-trough beach profile with an average slope of 0.025. 15 Pressure transducer sensors with the accuracy of order $\pm 1 \mathrm{~mm}$ were installed in 12 different locations along the flume (see Figure 4). The sensors were calibrated in situ and observed pressure readings were converted to surface elevations using Nielsen's (1983) local approximations (cf. Jafari et al., 2011). The statistical wave properties were calculated using spectral and zero-up crossing analysis and the incident wave properties for model boundary condition were extracted from the offshore sensor, i.e. the first sensor from the left hand side (see Figure 4). 36 different test scenarios of irregular wave conditions were conducted with $H_{r m s}$ varying from $0.02 \mathrm{~m}$ to $0.058 \mathrm{~m}$ and $T_{p}$ ranging from $0.623 \mathrm{~s}$ to $4.101 \mathrm{~s}$ corresponding of $\xi_{o}$ in the range of 0.7 to 2.4 . The irregular wave field was generated based on the JONSWAP spectrum with spectral shape factor of 3.3. More details of the laboratory tests were presented by Jafari and Cartwright (1999).
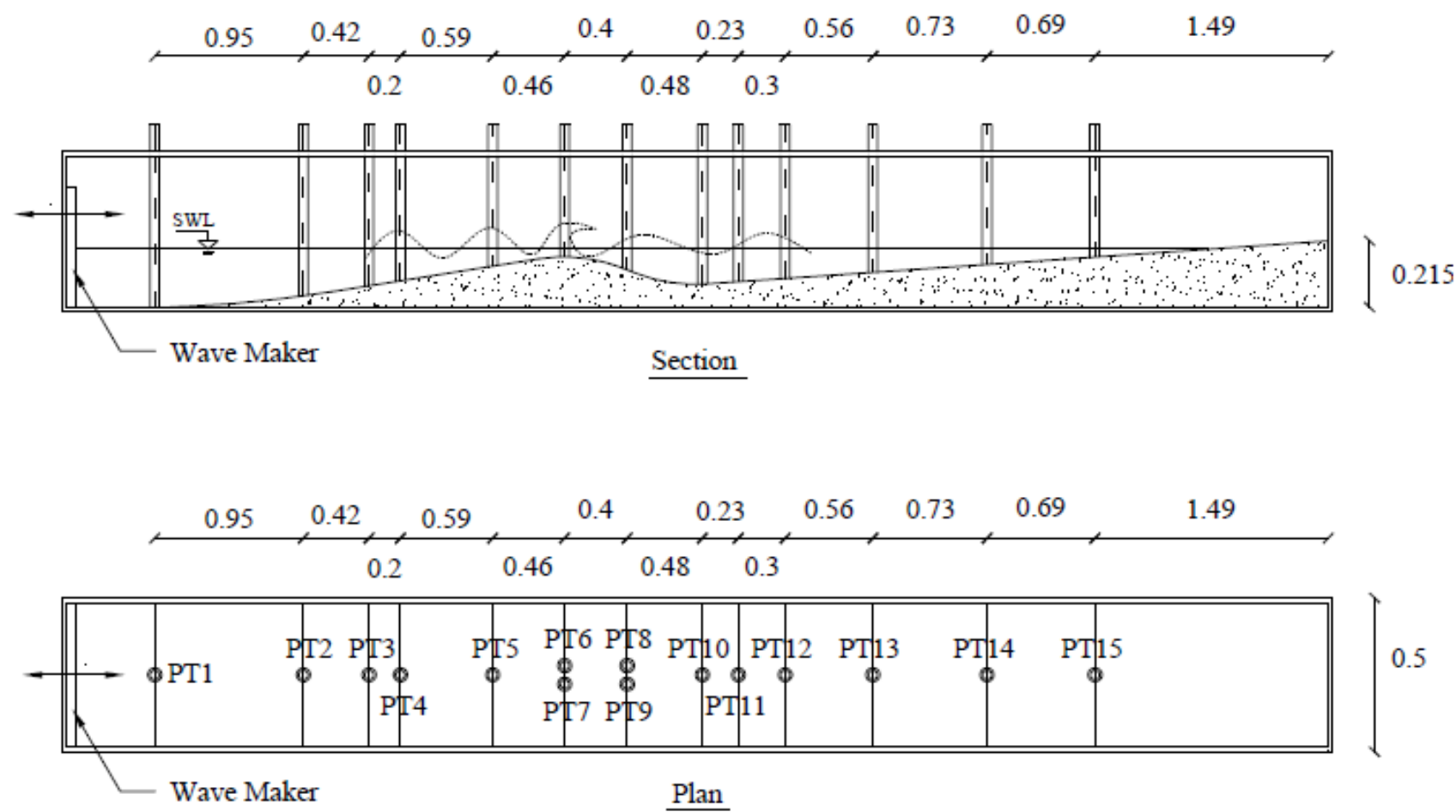

Figure 4. Elevation (top) and plan (bottom) views of the experiment setup in the wave flume. The wave maker is located at the far end of the left side of the flume. The pressure transducer sensor spacing are shown in both the plan and the section views of the wave flume 


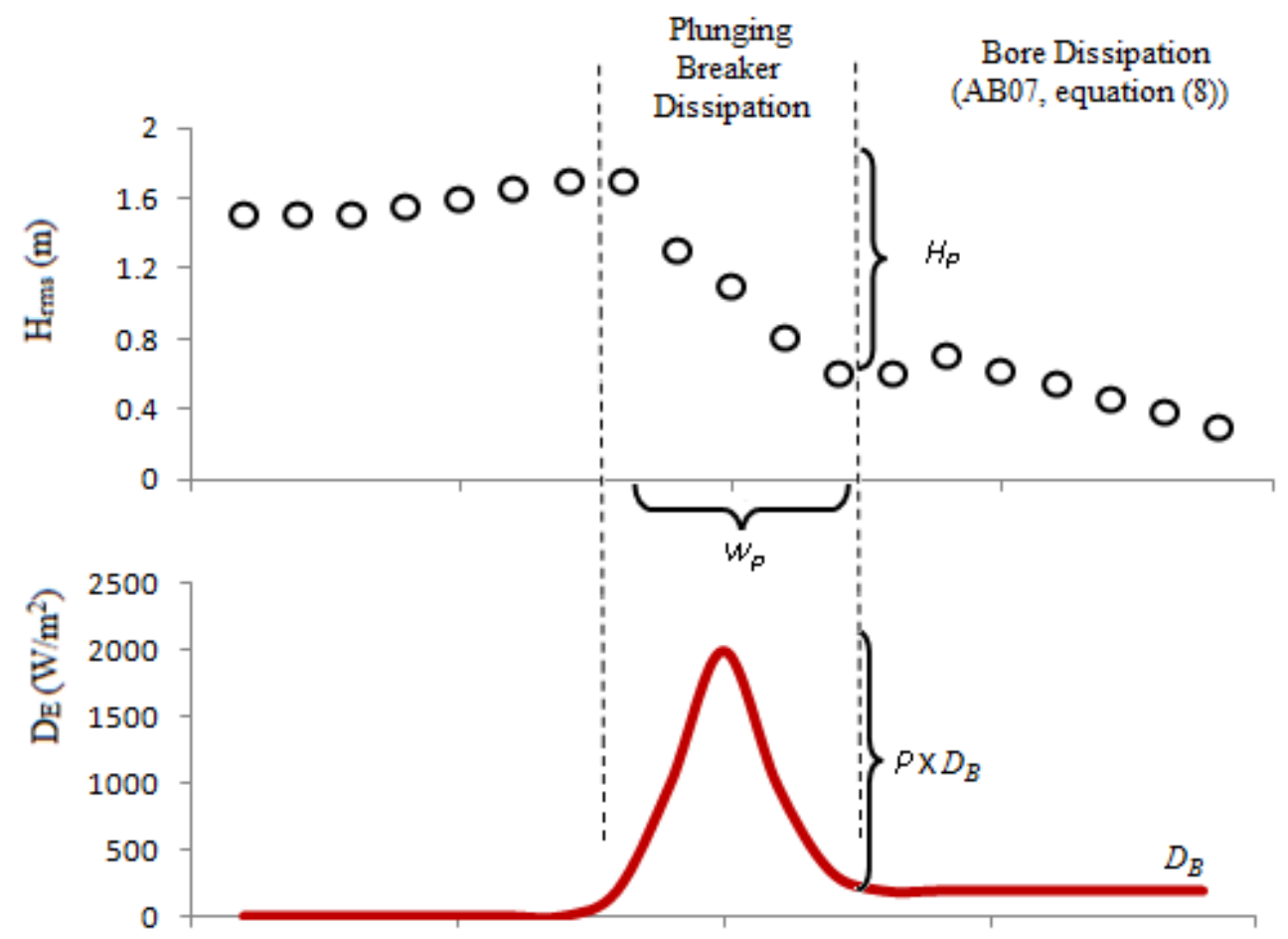

Figure 5. Schematic sketch of wave height change $\left(H_{P}\right)$ due to plunging break and the plunging width $\left(w_{p}\right)$ (top) and the amount of energy dissipation $(P)$ due to plunging (bottom)

\subsection{Additional Energy Dissipation due to Plunging breakers}

Figure 5 illustrates the present hypothesis that (a) due to the presence of plunging type breakers there is an additional spike in energy dissipation (P) at the break point and (b) this spike in energy dissipation occurs over a width $\left(w_{p}\right)$. The following outlines the extraction of these two quantities from the laboratory data.

In order to cover the gap of over prediction of the models, the plunging dissipation should be added to the dissipation term.

$$
\frac{\partial\left(E C_{g}\right)}{\partial x}=-D_{E}=\left(-D_{b}-D_{P}\right) \times Q_{b}=-P \times D_{B}
$$

where $P$ is the ratio of total energy dissipation over bore dissipation, $D_{E} / D_{B}$. In this case, $D_{E}$ was calculated based on the laboratory results by measuring the wave height before and after the breaking point and $D_{B}$ was computed followed by $\mathrm{AB} 07$ approach (cf. equation (8)). Froude numbers of all tests were calculated and all the test conditions were categorized as sub critical, i.e. $F r<1$. Additionally, dimensional analysis showed that all the field measurement conditions were sub critical as well. Therefore, it is reasonable to use the laboratory data for extracting the plunging dissipation 
term and apply it for modelling the prototype dissipation. Thus, the first step is to identify the parameters that govern the plunging dissipation.

$$
P=f\left(L_{b}, H_{b}, h_{b}, \tan \beta\right)
$$

Rearranging in to dimensionless terms, equation (11) becomes,

$$
P=f_{l}\left(\frac{\tan \beta}{\sqrt{H_{b} / L_{b}}}\right) \times f_{2}\left(\frac{H_{b}}{h_{b}}\right)
$$

where the $\tan \beta \times\left(H_{b} / L_{b}\right)^{-0.5}$ is the Iribarren number representative of surf similarity which indicates the type of breaking; and $H_{b} / L_{b}$ is ratio of breaking wave height over water depth which is usually shown in the literature as $\gamma_{b}$. Also, according to the laboratory data there is a strong linear correlation between offshore wave steepness, $\left(S_{o}=H_{d} / L_{o}\right)$ and the wave steepness at breaking point $\left(S_{b}=H_{b} / L_{b}\right)$. Hence, considering the fact that surf similarity is the bed slope over root square of wave steepness, the relation between offshore surf similarity, $\xi_{o}$, and surf similarity at break point, $\xi_{b}$, is defined as:

$$
\xi_{b}=\frac{1}{\sqrt{2.92}} \xi_{o}=0.585 \xi_{o} \quad \text { which } R^{2}=0.997
$$

Considering equation (13), the plunging coefficient function can be rewritten as follows:

$$
P=f_{3}\left(\xi_{o}\right) \times f_{2}\left(\gamma_{b}\right)
$$

Experimental data showed that the best fit curves for $f_{2}\left(\gamma_{b}\right)$ and $f_{3}\left(\xi_{o}\right)$ are linear and power, respectively. The $P$ factor was calculated from the actual plunging dissipation values which were extracted from the experimental data by numerically evaluating equation (1) for $D_{E}$ using the measured $\underline{H}_{r m s}$ just before and just after breaking and then normalizing over the bore dissipation calculated using equation (5). Moreover, based on the observations, for $\xi_{o}>0.4$, the breaker type is more of the plunging. Therefore, only data extracted from test cases with $\xi_{o}>0.4$ were considered for the curve fitting process. Afterwards, offshore surf similarity, $\xi_{o}$, was plotted against normalized plunging dissipation over $\gamma_{b}$ and best fit was achieved for $f_{3}$ (see Figure 6). Hence, the plunging correction function, based on experimental data was achieved as follows,

$$
P=\gamma_{b} \times\left(1.442 \times \xi_{o}^{3.678}+7.552\right)
$$

As a result, if the breaker type is more of the plunging form rather than spilling, the dissipation term in the right hand side of the equation (10) can be calculated as follows, 


$$
D_{E}=D_{B} \times \gamma_{b} \times\left(1.442 \times \xi_{o}^{3.678}+7.552\right)
$$

where, $D_{B}$ is the total amount of dissipation calculated by multiplying single bore dissipation, $D_{b}$ (see equation (5)), to the ratio of breaking waves over total number of waves, i.e. $Q_{b}$. In this research, the applied $D_{B}$ is what Alsina and Baldock (2007) recommended for bore dissipation (equation (8)).

In plunging breakers, after impact of the first jet, large scale vortices extend downward the breaking point and the dissipation occurs throughout the plunging width (Battjes, 1988). In order to find a plunging width equation, a two-step process is required. The first step is to consider the relevant parameters and then reduce the relationship to an equation with less independent parameters

$$
w_{p}=f\left(L_{o}, H_{o}, \tan \beta, T_{p}\right)
$$

The second step is to decide on the quantitative form of the dimensionless relation based on the measurement of plunging depth (Nielsen, 1989). Reasonable candidates for normalized plunging width are offshore wave steepness and surf similarity.

$$
\frac{w_{p}}{L_{o}}=f_{4}\left(\xi_{o}\right) \times f_{5}\left(s_{o}\right)
$$

Measured data showed that the relation of offshore surf similarity, $\xi_{o}$, and $w_{p} / L_{o}$ is in order of one. Then the following form of the $f_{5}$ was deduced and this was followed by performing extensive curve fitting over the experimental data (see Figure 7)

$$
\frac{w_{p}}{L_{o} \times \xi_{o}}=f_{5}\left(s_{o}\right)
$$

Consequently the best relationship for calculating the plunging width is given as follows,

$$
w_{p}=7509 \times L_{o} \times \xi_{o} \times s_{o}^{1.873}
$$

\subsection{Shoaling}

Theoretically equation (1) should capture the shoaling in the cases where the energy dissipation term on the right hand side of the equation is negligible and the changes in the bathymetry cause an increase in wave height due to the energy flux gradient. However, comparison of the data to model prediction shows that in most cases, models are unable to capture the actual amount of the shoaling. For example, as depicted in the top panel of Figure 8, the data show that the wave shoaled as the wave progressed to the point of the offshore bar knoll, and then breaking occurred. None of the models predicted shoaling properly. 
Therefore, the effect of the shoaling in the laboratory measurement is far larger than what equation (1) yields. The limitations of the underlying linear wave theory are the main suspects in this regard, however a detailed investigation of whether use of a higher order wave theory would improve this is beyond the scope of the present study. In the present context a numerical correction to improve shoaling in the models is developed.

Considering the number of breaking waves over the total number of waves in the distribution, $Q_{b}$, (see second panel of Figure 8), it can be a fair indicator for applying the shoaling into the model. Where $Q_{b}$ is close to zero, wave profile is subjected to shoal (Nielsen, 2009) however, for small values of $Q_{b}$ the resulting energy dissipation is still large enough to significantly reduce shoaling. In order to improve the model to predict the shoaling in the same order as laboratory measurements, the small values of $Q_{b}$ were neglected. The threshold value of $Q_{b}$ was considered $5 \%$ in order to solve equation (1) just for shoaling, i.e. for $Q_{b} \leq 0.05$ right hand side of equation (1) was set equal to zero. The value of $5 \%$ was determined via numerical calibration of the models to obtain a better model-data comparison in the shoaling region. The $Q_{b}$ in present work is calculated as suggested by B98 approach.

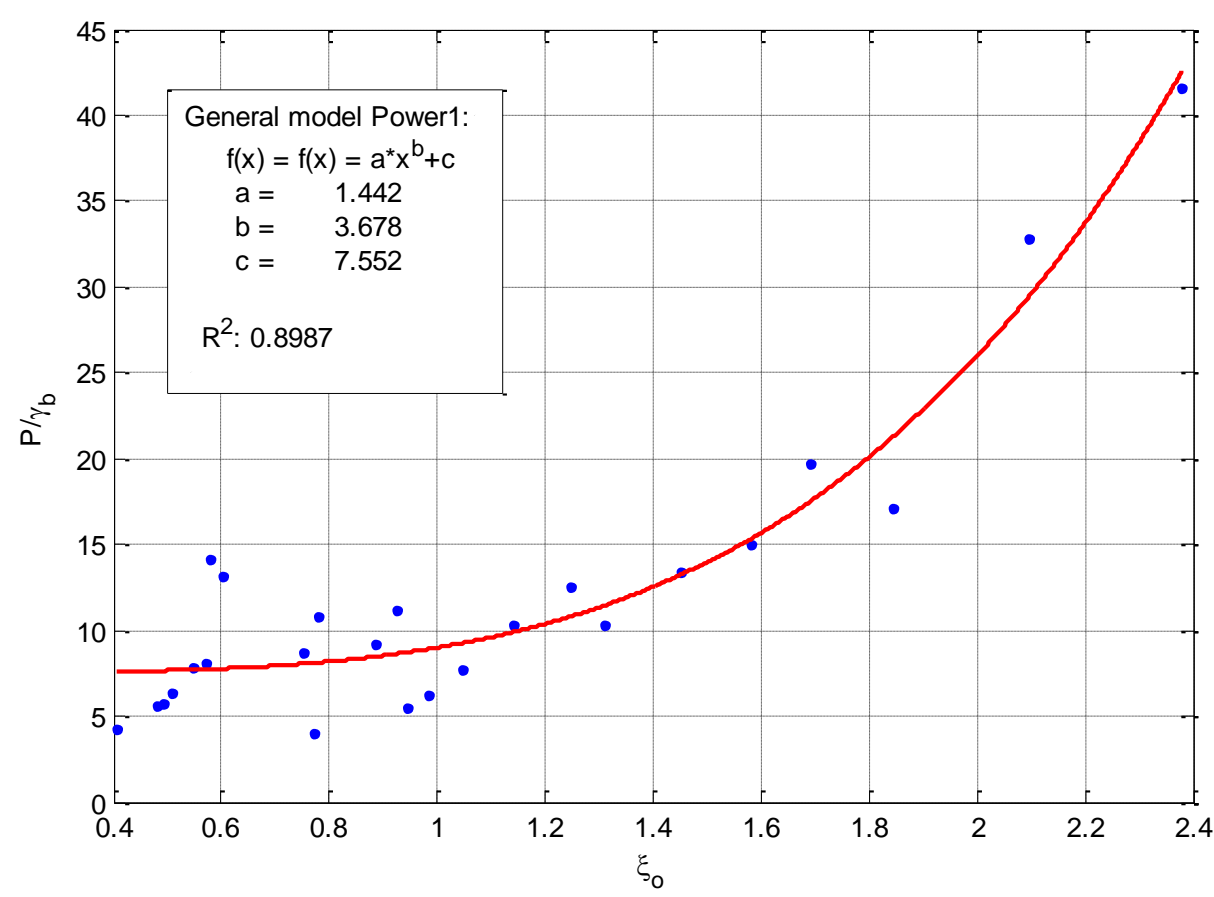

Figure 6. Curve fitting $f_{3}\left(\xi_{o}\right)$ against $P / \gamma_{b}$ using experimental data 


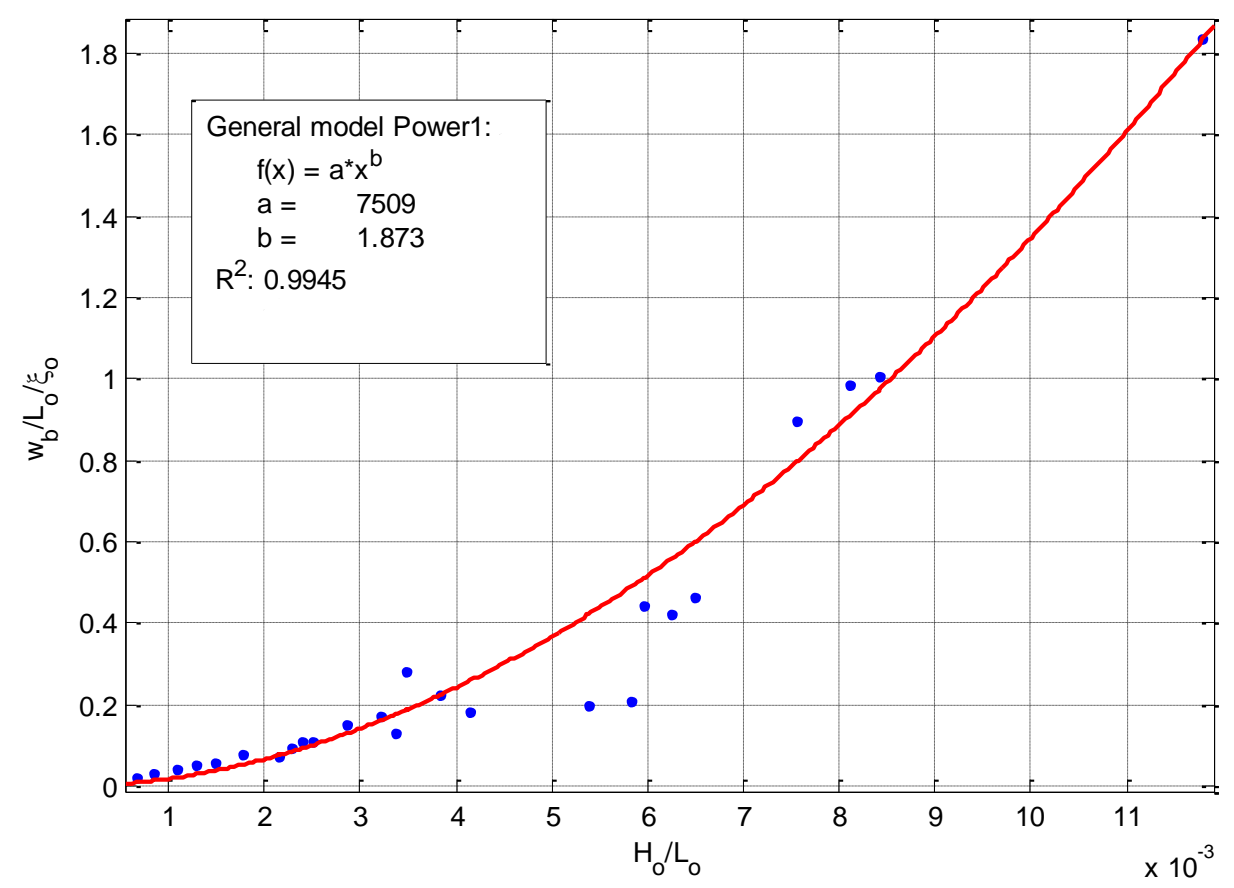

Figure 7. Curve fitting $f_{5}\left(s_{o}\right)$ against $w_{p} / L_{d} / \xi_{o}$ using experimental data
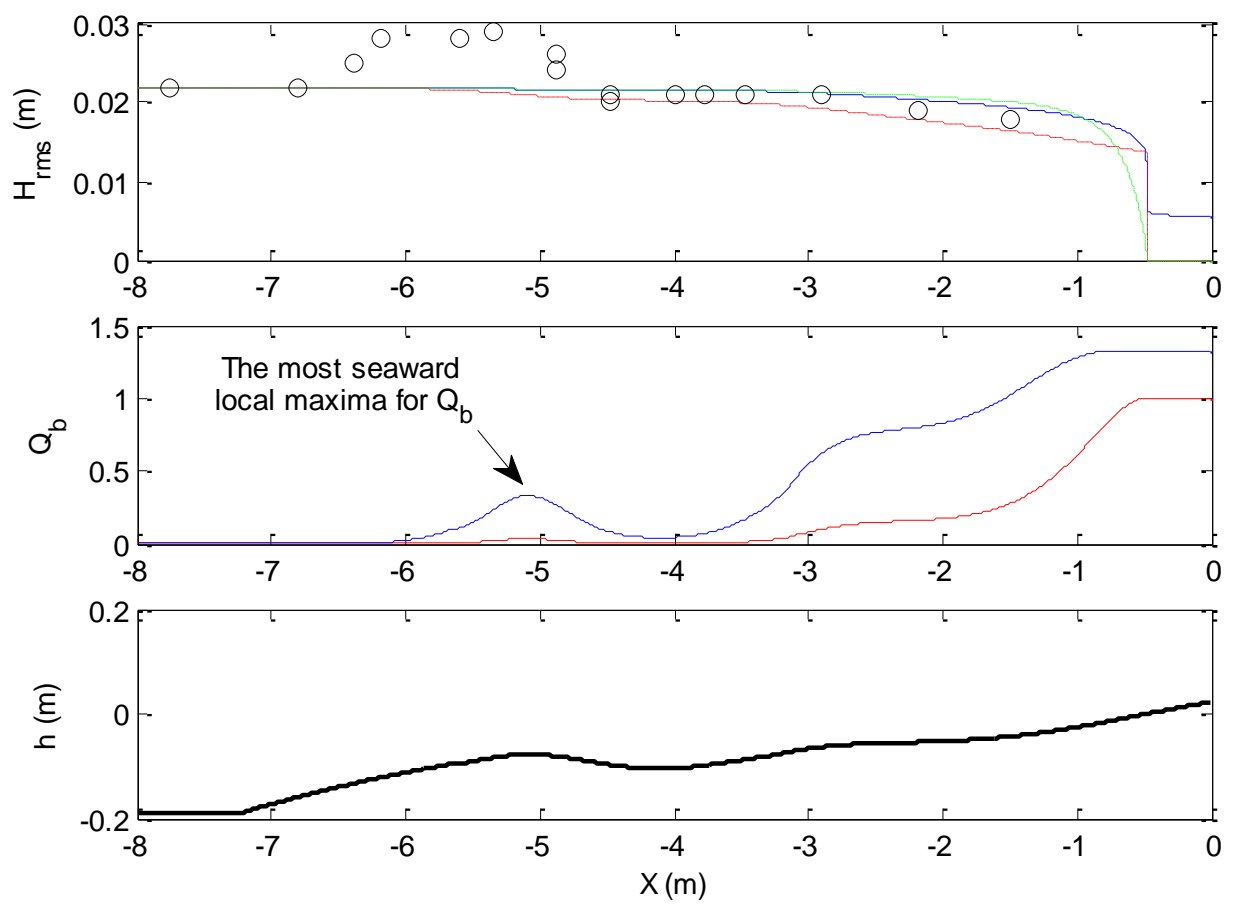

Figure 8. Defining the breaking point using turning point in $Q_{b}$. Top panel: $H_{r m s}$ distribution of lab data (circles) which $\mathrm{L}_{0}=26.26, H_{m s o}=0.02, T_{z}=2.55$ against model prediction of AB07 (blue solid line), B98 (red dashed line) and TG83 (green dotted line); 2nd panel is $Q_{b}$ distribution of AB07 (blue line), B98 (red line) and 3rd panel is the beach profile. 


\subsection{Breaking point}

One of the most critical parameters in implementing the new plunging dissipation term into the model is to determine the breaking point location. Again, $Q_{b}$ can be a useful indicator. This means that where waves approach to the head of the offshore bar, $Q_{b}$ starts to increase as more waves in the distribution start breaking (see Figure 8). As it can be seen in the second panel of Figure 8, right at the breaking point, at about $5 \mathrm{~m}$ offshore, $Q_{b}$ reached to its relative maximum value which is adopted here as a proxy for breaking point location.

\subsection{Model implementation}

Wave energy is propagated from offshore by the conservation of energy equation until the break point is reached at which time the new plunging breaker energy dissipation term is switched on and off according to the procedure outlined further below. After the breakpoint, the energy dissipation is modelled using the existing bore dissipation term of AB07.

The switching on and off of the plunging breaker term is controlled by the following procedure:

1. Calculate $Q_{b}$ using approach of $\mathrm{B} 98$ for each cell and if $\mathrm{Q}_{\mathrm{b}}<5 \%$ then set $\mathrm{D}_{\mathrm{E}}=0$. This is to ensure that shoaling is adequately captured (cf. section 4.3)

2. Define the breakpoint location by identifying the most seaward local maximum in $\mathrm{Q}_{\mathrm{b}}$ (cf. Figure 8)

3. Determine the type of breaking (plunging or spilling) by calculating the offshore surf similarity parameter for each cell

4. If the breaker type was defined as plunging, $D_{E}$ is calculated based on equation (16) over the breaker width defined by equation (20) after which energy dissipation is modeled using the bore dissipation approach of $\mathrm{AB} 07$.

This model, hereafter is called JC13, was run for the cases that the initial breaking is of the plunging type.

\subsection{Results}

The improvement of the modified AB07 model (JC13) against laboratory data is depicted in Figure 9 for a case where the offshore surf similarity is close to 0.5 , which is the boundary between spilling and plunging breaker types. Also Figure 10 represents an improvement in the model for a larger value of $\xi_{o}$ which was 2.095 in this case. Both figures clearly show that the JC13 model captures the shoaling before the breaking point ( $\mathrm{x} \sim-7 \mathrm{~m}$ to $-5 \mathrm{~m}$ in Figure 9 and $\mathrm{x} \sim-6 \mathrm{~m}$ to $-5 \mathrm{~m}$ in Figure 10) as is 
indicated by the laboratory data. Further, the sudden drop in the wave height due to plunging breaking in the data is also determined by the JC13 model.

To quantify each model's overall predictive ability, the coefficient of determination, $R^{2}$, and relative root mean square error in prediction, $\varepsilon$, as used by Alsina and Baldock (2007) and calculated as follows:

$\varepsilon=\frac{1}{N} \frac{\sqrt{\sum\left(H_{\text {comp }}-H_{\text {meas }}\right)^{2}}}{H_{o}} \times 100$

where, $N$ is the total number of samples and $H_{o}$ is the offshore boundary wave height. Also, the statistical wave height characteristics that is used here is the root mean square wave height, $H_{r m s}$. Figure 11 shows the model error, $\varepsilon$, versus offshore surf similarity, $\xi_{o}$. In just one case the calculated error of JC13 prediction was marginally higher than those of previous models (5\% higher than AB07 and $4 \%$ higher than B98 and TG83 for test with $\xi_{o}=0.82$ ). This was due to the fact that, in that case, the break point which was determined by the model was not exactly in the same spot as the data. Hence, the root mean square error calculated of the differences between the model and measurement was increased about 5\% more than what was obtained from the previous models.

The improvement in the JC13 prediction for the field measurement is presented in Figure 12 for a data block of ECL. The observations showed that the criteria of defining breaker type is less than what is recommended in Coastal Engineering Manual (Smith, 2002). $\xi_{o}$ was calculated for all the recorded field data. The results showed that basically all the calculated $\xi_{o}$ were less than 0.5 , which based on Coastal Engineering Manual (Smith, 2002) the breaker should be of the spilling type. However, the dominant breaker type based on visual observation was of the plunging. Consequently, based on the shape of the recorded wave height profile across the surfzone, and the associated value of $\xi_{o}$, the boundary of the breaker type i.e. spilling to plunging was modified. As a result, $\xi_{o}$ equal to 0.15 was chosen as a local criterion of determination of the breaker type based on Southeast Queensland recorded data. This value was adopted in JC13 for field data. JC13 along with the previous models were run against all the data blocks recorded from the field measurements. The accuracy of model prediction against recorded field data was measured by calculating the error, $\varepsilon$, for each data block and results were depicted in Figure 13. The calculated error, $\varepsilon$, for all the cases was improved using JC13. 

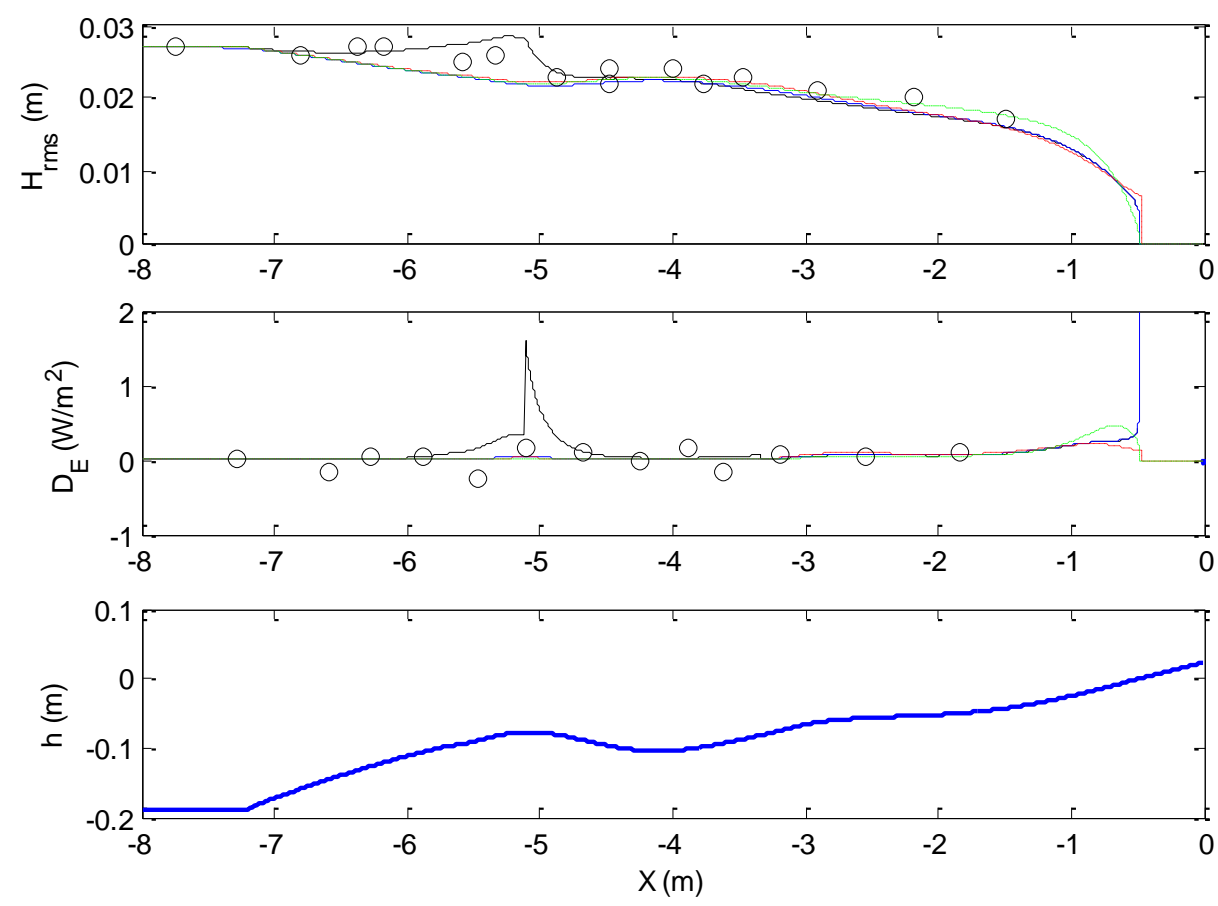

Figure 9. Model-data comparison for laboratory measurements with $\xi_{o}=0.537$. Data (circles), JC13 (black solid line), AB07 (blue solid line), B98 (red dotted line) and TG83 (green dash line); top panel is $H_{r m s}$ distribution, 2nd panel is distribution of energy dissipation per unit area and 3rd panel is the beach profile
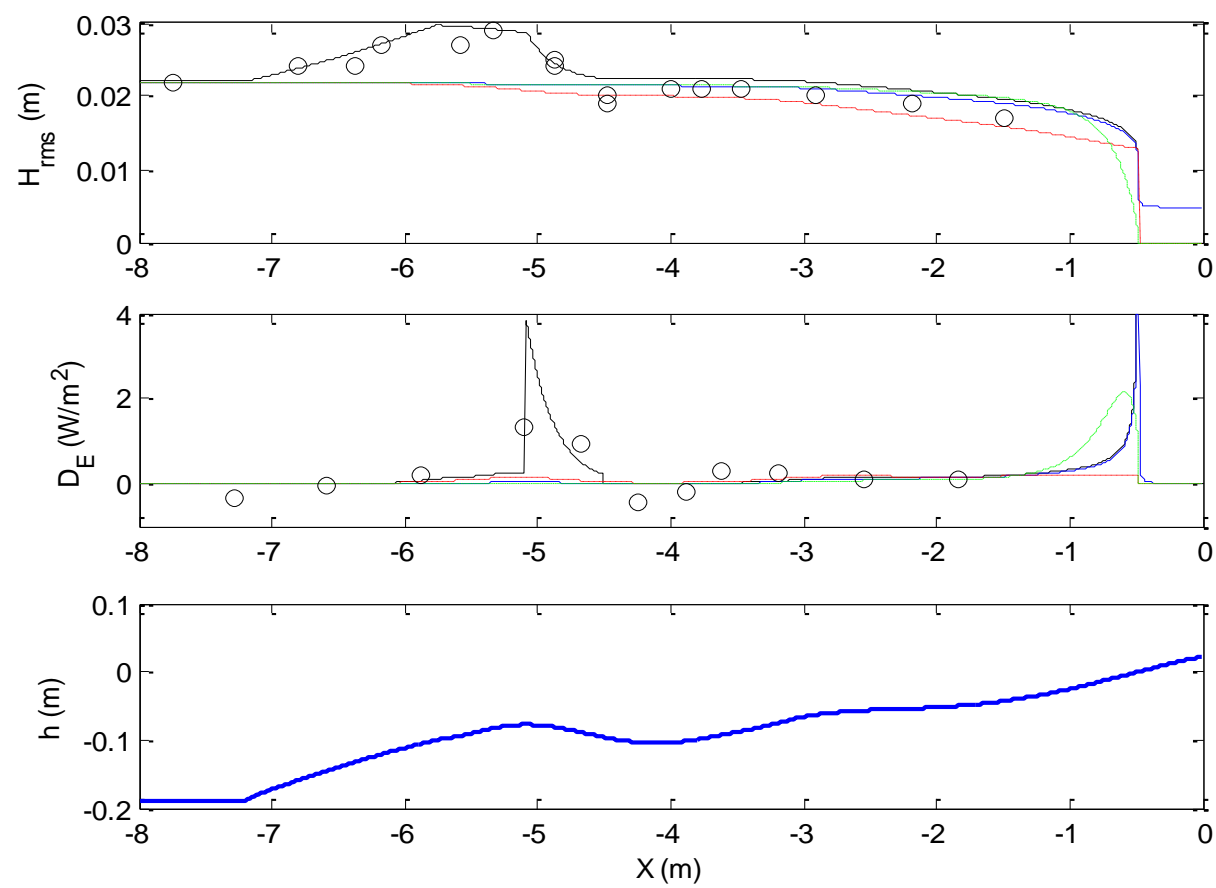

Figure 10. Laboratory data distribution along surfzone (circles) $\xi_{o}=2.095$, against model prediction of JC13 (black solid line), AB07 (blue solid line), B98 (red dotted line) and TG83 (green dash line); top panel is $H_{r m s}$ distribution, 2nd panel is distribution of energy dissipation per unit area and 3rd panel is the beach profile 


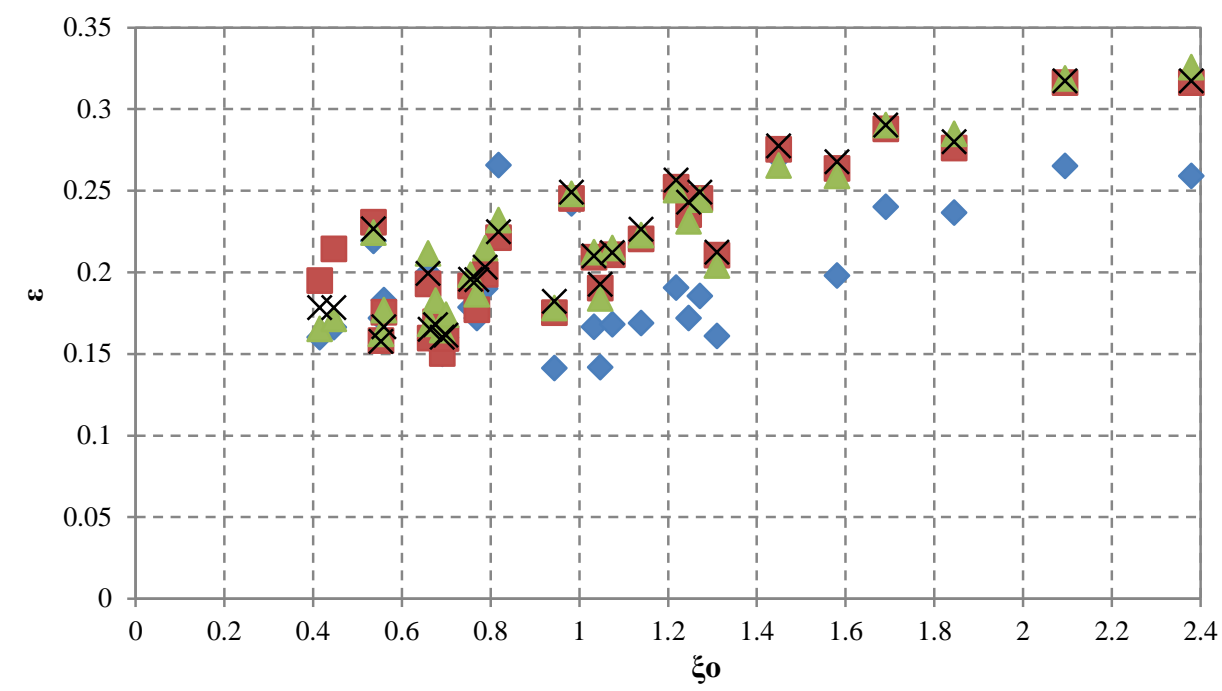

Figure 11. Calculated error of models prediction based on equation (21) against all the laboratory measurements versus $\xi_{\mathrm{o}}$, JC13(•), AB07 (•), B98( $\left.\mathbf{\Delta}\right)$, TG83( $\times$ )
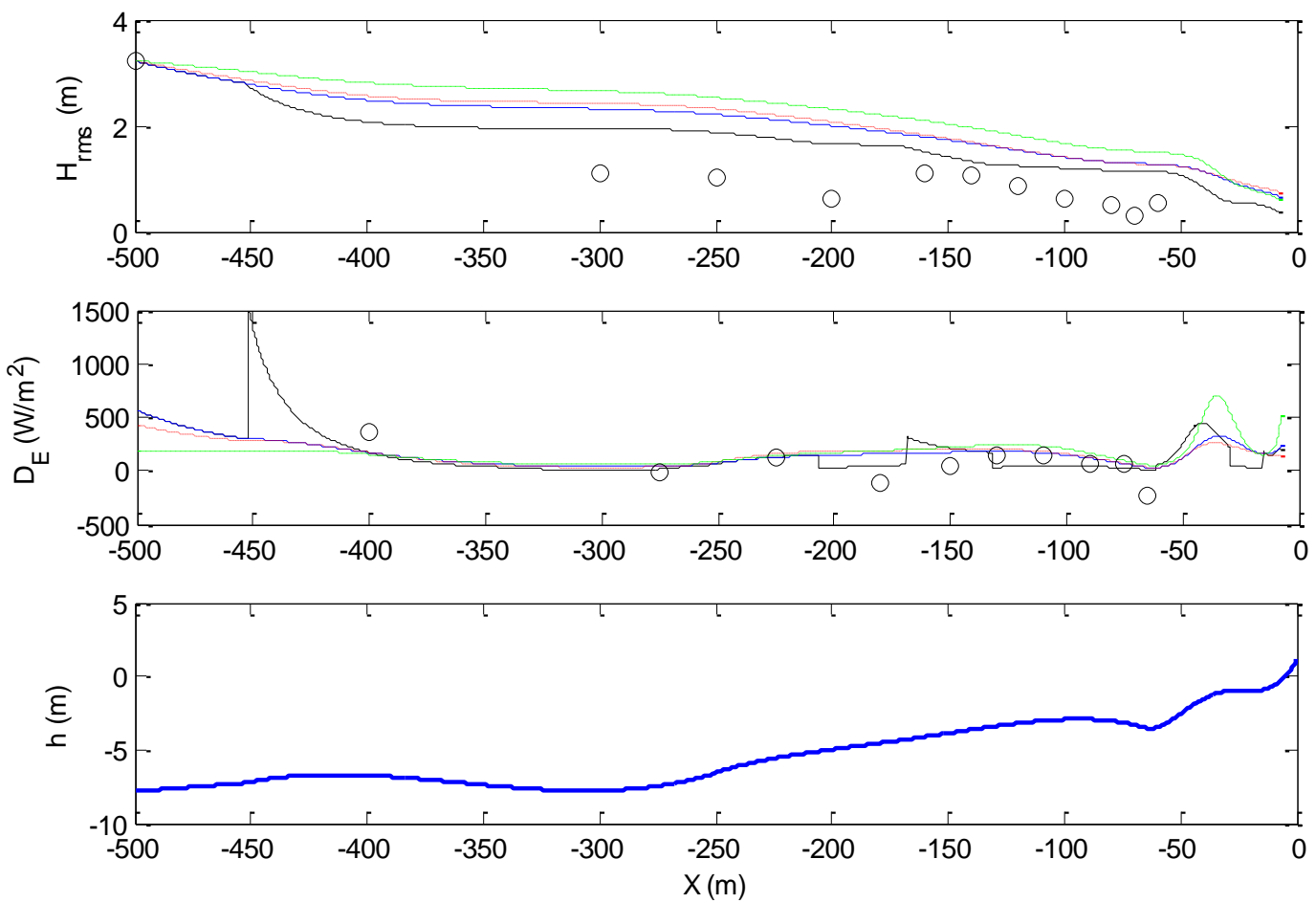

Figure 12. ECL measured data distribution along surfzone (circles) $\xi_{o}=0.216$, against model prediction of JC13 (black solid line), AB07 (blue solid line), B98 (red dotted line) and TG83 (green dash line); top panel is $H_{r m s}$ distribution, 2nd panel is distribution of energy dissipation per unit area and 3rd panel is the beach profile 


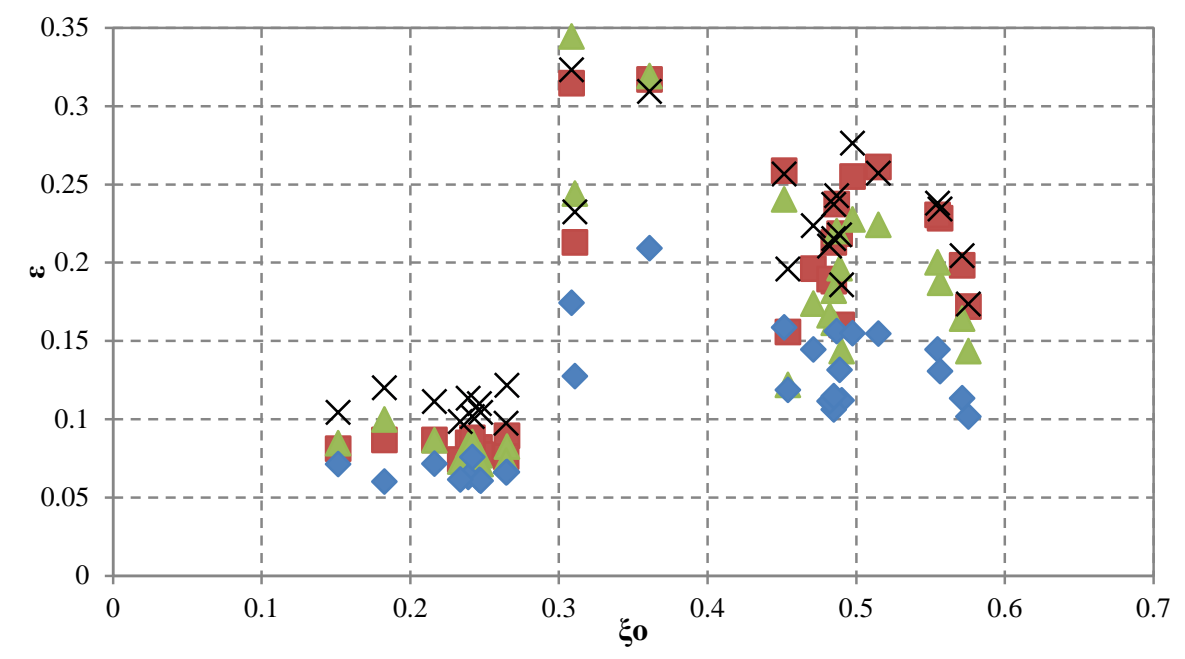

Figure 13. Calculated error of models prediction based on equation (21) against all field measurements versus $\xi_{0}, \mathrm{JC} 13(\bullet), \mathrm{AB} 07(\boldsymbol{\bullet}), \mathrm{B} 98(\boldsymbol{\Delta})$, TG83( $\times$ )

\section{Conclusion}

Predictions of parametric wave transformation models based on the bore dissipation model were compared field measurements and were unable to capture the amount of dissipation at the breakpoint in the cases where plunging type breakers dominated. Consequently, new empirical functions quantifying the energy dissipation associated with plunging breakers were extracted from extensive laboratory measurements covering a range of offshore surf similarity parameter values, $\xi_{o}$, from 0.4 to 2.4 .

A new empirical energy dissipation formula was developed as a function of offshore surf similarity, $\xi_{o}$, and ratio of wave height over water depth at the breaking point, $\gamma_{b}$. In addition, an experimental relationship for plunging break zone width, $w_{p}$, was extracted from laboratory data based on offshore surf similarity, $\xi_{o}$, and offshore wave steepness, $S_{o}$.. Implementation of the new expressions into the AB07 model resulted in a significant improvement in the wave height profile prediction across the surfzone.

\section{Acknowledgements}

The help and assistance of Mr. Christopher Williams through performing the laboratory test is appreciated. The buoy data was obtained from the Queensland Department of Environment and Resource Management. Funding for the present work and financial support for the first author has been provided by Australian Research Council grant number DP0877235.

\section{References}

Alsina, J.M. and Baldock, T.E., 2007. Improved representation of breaking wave energy dissipation in parametric wave transformation models. Coastal Engineering 54 765-769. 
Baldock, T. and Simmonds, D., 1999. Separation of incident and reflected waves over sloping bathymetry. Coastal Engineering, 38(3): 167-176.

Baldock, T.E., Holmes, P., Bunker, S. and Van Weert, P., 1998. Cross-shore hydrodynamics within an unsaturated surf zone. Coastal Engineering, 34: 173-196.

Battjes, J., 1988. Surf-zone dynamics. Annual Review of Fluid Mechanics, 20(1): 257-291.

Battjes, J.A. and Janssen, J.P.F.M., 1978. Energy loss and set-up due to breaking of random waves, 16th Int. Conf. on Coastal Engineering ASCE, Hamburg, Germany, pp. 569-587.

Guard, P.A. and Baldock, T.E., 2007. The influence of seaward boundary conditions on swash zone hydrodynamics. Coastal Engineering, 54(4): 321-331.

Hunt, I.A., 1959. Design of seawalls and breakwaters. Journal of the Waterways and Harbors Division, ASCE, Vol. 85, No. WW3: 123-152.

Jafari, A. and Cartwright, N., 2012. Evaluation of a parametric-type wave transformation model against field and laboratory data. 33rd International Conference of Coastal Engineering, Santander, Spain, p.^pp. waves.51. doi:10.9753/icce.v33.waves.51.

Jafari, A., Cartwright, N. and Nielsen, P., 2011. Stormy wave analysis based on recorded field data on south-east coasts of queensland, australia. Journal of coastal Research, SI 64: 527-533.

Janssen, T. and Battjes, J., 2007. A note on wave energy dissipation over steep beaches. Coastal Engineering, 54(9): 711-716.

LeMehaute, 1962. On non-saturated breakers and the wave run-up. 8th International Conference of Coastal Engineering, New York, p.^pp. 77-92.

Nairn, R.B., 1990. Prediction of cross-shore sediment transport and beach profile evolution, University of London, London, England.

Nielsen, P., 1989. Analysis of natural waves by local approximations. Journal of Waterway, Port, Coastal, and Ocean Engineering, 115(3): 384-396.

Nielsen, P., 2009. Coastal and estuarine processes, 343. World Scientific.

Raubenheimer, B., Guza, R.T. and Elgar, S., 1996. Wave transformation across the inner surf zone. Journal of geophysical research, 101: 25,589-25,597.

Ruessink, B.G., Walstraa, D.J.R. and Southgate, H.N., 2003. Calibration and verification of a parametric wave model on barred beaches. Coastal Engineering, 48: 139-149

Smith, J.M., 2002. Coastal engineering manual, Chapter 4, Surf Zone Hydrodynamics, EM 1110-21100. U.S. Army Corps of Engineers, Washington, DC.

Svendsen, I.A., 2006. Introduction to nearshore hydrodynamics. Advanced series on ocean engineering, 24. World Scientific, $722 \mathrm{pp}$.

Thornton, E.B. and Guza, R.T., 1983. Transformation of wave height distribution. Journal of geophysical research, 88: 5925-5938.

van Rijn, L.C. et al., 2003. The predictability of cross-shore bed evolution of sandy beaches at the time scale of storms and seasons using process-based profile models. Coastal Engineering, 47(295-327). 